\title{
Über das Auftreten des „blauen“ Spektrums von Benzaldehyd
}

\author{
Von H. Schüler und L. Reinebeck \\ Aus der Forschungsstelle für Spektroskopie in der Max-Planck-Gesellschaft, Hechingen \\ (Z. Naturforschg. 5 a, 448-451 [1950]; eingegangen am 7. August 1950)
}

\begin{abstract}
Auf Grund neuerer Versuche wird gezeigt, daß man bei Benzol und verschiedenen Benzolderivaten eine Verunreinigung durch Benzaldehyd' noch bei einem Mischungsverhältnis von 1:1000000 in der Glimmentladung nachweisen kann.

Der Nachweis erfolgt durch das sogenannte „blaue“ Spektrum, das der $\mathrm{C}=\mathrm{O}$-Anregung des Benzaldehydmoleküls zugeschrieben wird und nicht der Anregung des freien Phenylradikals, wie es bisher von uns zur Diskussion gestellt wurde ${ }^{1}$.

Die Ähnlichkeit dieses Spektrums mit dem Phosphoreszenzleuchten, auf die früher ${ }^{2}$ hingewiesen wurde, zwingt zu zusätzlichen Annahmen bei der Deutung der Phosphoreszenzerscheinungen.
\end{abstract}

$\mathrm{B}$ ei den Untersuchungen von Benzolderivaten durch Elektronenstoß in der Glimmentladung wurden u. a. zwei Spektren im Sichtbaren beobachtet, die bei Versuchen mit verschiedenen Substanzen immer wieder in Erscheinung traten ${ }^{3}$. Wegen der Lage ihrer Hauptintensitäten wurden die Spektren als „grünes“ bzw. als „blaues“ Spektrum bezeichnet. Dabei zeigte sich zunächst, daß das „blaue“ Spektrum beim Benzaldehyd allein auftrat (kein „grünes"), während bei anderen Benzolderivaten beide Arten von Spektren beobachtet wurden. Es stellte sich heraus, daß das Erscheinen dieser Spektren von den Entladungsbedingungen abhing, und zwar wurde bei geringerem Substanzdampfdruck nur das "grüne“ und bei höherem Dampfdruck nur das „blaue“ Spektrum festgestellt (z. B. bei Äthylbenzol und $n$-Propylbenzol). Damit schien der Verdacht, daß es sich um Verunreinigungen handeln könnte, ausgeschlossen, denn bei einer Verunreinigung sollte man erwarten, daß sie unabhängig vom Dampfdruck immer in Erscheinung tritt.

Es seien zunächst nur die Beobachtungen am „blauen“ Spektrum diskutiert ${ }^{1}$.

Während bei den ersten Versuchen die Entladun-

1 H. S chüler u. L. Reinebeck, Z. Naturforschg. 4a, 577 [1949]. Das „blaue“ Spektrum liegt im Wellenlängengebiet $3700-5400 \AA$. Die Hauptbanden: $3713,0(\mathrm{~F}) ; 3970,3(\mathrm{E}) ; 4263,0(\mathrm{D}) ; 4597,4(\mathrm{C}) ; 4985,4(\mathrm{~B})$; 5420,3 (A) Å zeigen Schwingungsdifferenzen von $\Delta v=1743$; $1731 ; 1704 ; 1692 ; 1610 \mathrm{~cm}^{-1}$.

Das „blaue“ Spektrum war beobachtet bei: $\mathrm{C}_{6} \mathrm{H}_{5} \cdot \mathrm{CH}_{3}$; $\mathrm{C}_{6} \mathrm{H}_{5} \cdot \mathrm{C}_{2} \mathrm{H}_{5} ; \quad \mathrm{C}_{6} \mathrm{H}_{5} \cdot \mathrm{C}_{3} \mathrm{H}_{7}(n$ und $i) ; \mathrm{C}_{6} \mathrm{H}_{5} \cdot \mathrm{CH}_{2} \mathrm{OH}$; $\mathrm{C}_{6} \mathrm{H}_{5} \cdot \mathrm{CH}_{2} \mathrm{CN} ; \mathrm{C}_{6} \mathrm{H}_{5} \cdot \mathrm{CH}_{2} \mathrm{Cl} ; \mathrm{C}_{6} \mathrm{H}_{5} \cdot\left[\mathrm{CH}_{2}\right]_{2} \mathrm{Cl} ; \mathrm{C}_{6} \mathrm{H}_{5} \cdot\left[\mathrm{CH}_{2}\right]_{3}$ $\mathrm{Cl} ; \mathrm{C}_{6} \mathrm{H}_{5} \cdot \mathrm{COOH} ; \mathrm{C}_{6} \mathrm{H}_{5} \cdot \mathrm{COOC}_{2} \mathrm{H}_{5}$ und $\mathrm{C}_{6} \mathrm{H}_{5} \cdot \mathrm{CHO}$ (Benzaldehyd). gen mit Benzoldampf kein „blaues“ Spektrum aufwiesen, erhielten wir bei späteren mit besonders hohem Dampfdruck auch Anzeichen von diesem Spektrum. Ein Umzug des Laboratoriums unterbrach die Versuche und bei Wiederaufnahme der Untersuchungen konnten wir beim Benzol auch unter extremen Druckbedingungen das „blaue“ Spektrum nicht mehr feststellen. Da beim Neuaufbau der Apparatur auch die Glaszuführungen zur Pumpe erneuert worden waren, bestand die Möglichkeit, daß vorher trotz vorgeschalteter Kühlfallen mit flüssiger Luft Spuren von Verunreinigungen in die Entladungsbahn hineindiffundiert sein konnten. Um das zu prüfen, wurde das Benzol, das also kein „blaues“ Leuchten zeigte, zusätzlich mit 1\% Benzaldehyd verunreinigt. Benzaldehyd wurde zugesetzt, da hier das „blaue“ Spektrum mit der größten Intensität auftrat (weitere Gründe s. weiter unten). Eine Aufnahme mit dieser Mischung ergab ein ebenso starkes „blaues“ Spektrum wie bei reinem Benzaldehyd, und zwar unabhängig vom benutzten Substanzdampfdruck. Bei weiterer Verdünnung (Mischungsverhältnisse $1: 10^{-4}$ und $1: 10^{-5}$ ) nahm die Intensität des Spektrums ab und bei einem Mischungsverhältnis Benzol zu Benzaldehyd von $1: 10^{-6}$ wurde das Spektrum so schwach, daß man es nur bei höherem Substanzdruck noch angedeutet fand. $1: 10^{-6}$ entspricht also offensichtlich dem Verunreinigungsgrad, der bei den ersten Versuchen mit Benzol vorgelegen haben könnte.

2 H. Schüler, A. Wo eldike u. L. Reine b e c k, Z. Naturforschg. 4a, 124 [1949].

3 Die beiden Spektren sind bei $\mathrm{H}$. S chüler u. A. W o eldike, Physik. Z. 43, 17 [1942], Tafel V, VI und VII, wiedergegeben. 
Diese Nachweisbarkeitsgrenze von $1: 10^{-6}$ liegt bei wesentlich geringeren Konzentrationen als die bei der Absorption, die wir bisher zur Prüfung der Reinheit unserer Substanzen benutzt haben. Absorptionsversuche in unserer Röhre mit Mischungen von Benzol und Benzaldehyd ließen zwar noch $1: 10^{-3}$, aber nicht mehr $1: 10^{-4}$ nachweisen.

Es ist verwunderlich, daß so schwache Verunreinigungen überhaupt noch in Emission nachweisbar sind. Die Erscheinung, daß Benzaldehyd als Verunreinigung von $1 \%$ ebenso stark leuchtet wie die reine Substanz, läßt auf eine ungewöhnlich große.Energieübertragung des Benzols auf das Benzaldehydmolekül schließen ${ }^{4}$.

Beim Toluol wurde das „blaue“ Spektrum immer leichter und stärker als beim Benzol beobachtet. Auch andere Autoren ${ }^{5}$ haben auf seine Anwesenheit bei höherem Substanzdruck hingewiesen. Es liegen jetzt aber Versuche mit der neuen Apparatur vor, wo selbst bei höherem Substanzdruck kein "blaues“ Spektrum mehr in Erscheinung tritt. Dagegen zeigte sich, daß beim Zulassen von geringen Mengen von Sauerstoff in den strömenden Toluoldampf während der Entladung das „blaue“ Spektrum intensiv auftritt, was auf eine chemische Reaktion, die wahrscheinlich zu Benzaldehydbildung führt, hindeutet. Das „blaue“ Spektrum verschwand nach einigen Minuten, nachdem offensichtlich der Sauerstoff aufgebraucht war. Bei dieser Reaktion müssen angeregte Molekülzustände maßgeblich beteiligt sein, da Toluol unter Normalbedingungen nicht mit molekularem Sauerstoff reagiert. Bei Benzol war eine solche Reaktion nicht festzustellen.

Eine vollständige Entfernung des „blauen“ Spektrums, das bei den anderen erwähnten Benzolderivaten beobachtet worden war $^{1}$, ist uns bisher noch nicht gelungen. Es kann sich auch hier um geringere Verunreinigungen mit Benzaldehyd als $1: 10^{-3}$ handeln,

4 Schon bei reinem Benzaldehyd ist es bemerkenswert, daß das Spektrum so außergewöhnlich intensiv beobachitet wird, während der Absorptionskoeffizient mit seinem Wert $\varepsilon=30$ auf einen sehr schwachen Übergang hinweist. Es muß also bei der Elektronenstoßanregung außer der direkten Besetzung, die der Absorption mit $\varepsilon=30$ entspricht, noch eine Auffüllung dieses angeregten Zustandes auf einem anderen Wege möglich sein.

Um diese Prozesse besser beurteilen zu können, haben wir eine Anordnung getroffen, die es gestattet, aus zwei getrennten Behältern zwei verschiedene organische Substanzen in die Entladungsröhre zu verdampfen. Die eine Substanz wird so temperiert, da $\beta$ sie die Entladung im Beobachtungsraum trägt, während der Dampf der anderen nur spurenweise zugesetzt wird. Über die Ergebnisse dieser Versuche soll an anderer Stelle berichtet werden. die wir in Absorption nicht mehr nachweisen können. Die chemischen Herstellungsmethoden garantieren im allgemeinen nicht den hier notwendigen extremen Reinheitsgrad.

Nach diesen Befunden zwingt das Auftreten des gleichen „blauen“ Spektrums bei verschiedenen Monoderivaten des Benzols nicht mehr dazu, ein gemeinsames Molekülbruchstück als Träger dieses Spektrums anzunehmen. Damit wird das Hauptargument unseres Vorschlages, das freie Phenylradikal als Ursache des „blauen“ Spektrums anzusehen, hinfällig; vielmehr ist jetzt wohl die Annahme berechtigt, daß bereits geringe Spuren von Benzaldehyd dafür verantwortlich sind. Unsere bisherige Auffassung wird dadurch verständlich, daß man experimentell keine Anhaltspunkte dafür hatte, daß bei Untersuchungen an einer verunreinigten Substanz in der Glimmentladung das Leuchten der Verunreinigung nur bei erhöhtem Substanzdampfdruck auftritt und bei geringerem überhaupt nicht zu beobachten ist. Es bleibt auch weiterhin ungeklärt, warum durch Druckänderungen das Spektrum der Verunreinigung beliebig hervorgerufen oder unterdrückt werden kann.

Wie bereits erwähnt, haben M c Vick e r, M a r s h und $\mathrm{S} \mathrm{t} u$ a $\mathrm{r} \mathrm{t}^{5}$ bei ihren Tesla-Lumineszenzversuchen das gleiche „blaue“ Spektrum gefunden, das sie den einzelnen Benzolderivaten selbst zuordneten.

Auf Grund von Fluoreszenzbeobachtungen hat $T$ e r e $n$ i n darauf hingewiesen, daß bei diesen Untersuchungen eine gemeinsame Verunreinigung durch Benzaldehyd, „gebildet durch chemische Reaktionen der angeregten Verbindungen “, vorliegen könnte ${ }^{6}$.

Die Gründe, warum wir gerade das Benzaldehydmolekül jetzt als Träger des „blauen“ Spektrums ansehen, sind folgende:

1. Wie schon erwähnt, tritt das Spektrum beim Benzaldehyd mit der größten Intensität auf und ist unabhängig vom Substanzdampfdruck.

2. Zwischen den Hauptbanden des Spektrums tritt eine Schwingungsfolge von $\Delta v \sim 1700 \mathrm{~cm}^{-1}$ auf ${ }^{1}$, die das ganze Spektrum beherrscht. Schwingungen dieser Größe sind vielfach beobachtet und werden der $\mathrm{C}=\mathrm{O}$-Gruppe zugeschrieben; sie treten z. B. auch im Emissionsspektrum des Formaldehyds auf.

3. Benzaldehyd besitzt bei Absorption im gasförmigen Zustand in der Gegend $3720-3160 \AA$ ein Bandenspektrum mit geringem Ảbsorptionskoeffizien-

5 W. H. M c Vicker, J. K. M a r s h, A. W. Stuart, J. chem. Soc. [London] 123 II, 2147 [1923].

6 s. P. Pring s h e i m, Fluorescence and Phosphorescence, Interscience Publ. Inc. New York, 1949, S. 270. 
ten ${ }^{7}$. Wie wir gezeigt haben, stimmt die langwelligste Hauptbande der Absorption ( $\lambda 3713 \AA$ ) mit der kurzwelligsten $(F)$ des „blauen“ Emissionsspektrums überein. Daraus ist zu schließen, daß Absorptions- und Emissionsspektrum dem gleichen Elektronenzustand angehören.

Wenn nun das „blaue“ Spektrum bei den Monoderivaten des Benzols auf eine gemeinsame Verunreinigung durch Benzaldehyd zurückgeführt wird, ist jetzt auch die Übereinstimmung des „blauen“ Spektrums von $p$-Xylol $\left[\mathrm{CH}_{3} \cdot \mathrm{C}_{6} \mathrm{H}_{4} \cdot \mathrm{CH}_{3}\right]$ und $p$-Toluylaldehyd $\left[\mathrm{CH}_{3} \cdot \mathrm{C}_{6} \mathrm{H}_{4} \cdot \mathrm{CHO}\right]$, das gegen dasjenige von Benzaldehyd etwas nach Violett verschoben ist ${ }^{3}$, nicht mehr als ein Beweis für ein gemeinsames Bruchstück (das freie $p$-Tolylradikal) aufzufassen, sondern es sollte sich auch beim $p$-Xylol, wo dieses Spektrum nur bei erhöhtem Dampfdruck erscheint, um eine geringfügige Verunreinigung mit $p$-Toluylaldehyd handeln. Versuche in dieser Richtung sprechen auch für eine solche Annahme, denn eine Beimischung von 1\% $p$-Toluylaldehyd zu Benzol zeigte stark das entsprechende „blaue“ Spektrum, während 1\% $p$-Xylol in Benzol kein „blaues“ Leuchten ergab.

$p$-Toluylaldehyd hat nun wie Benzaldehyd im gasförmigen Zustand in dem Gebiet von $3700-3150 \AA$ eine schwache Bandenabsorption. Da hier die gleiche Übereinstimmung der langwelligsten Absorptionshauptbande mit der kurzwelligsten Emissionsbande gefunden wurde, so dürfte es sich kaum um eine zufällige Koinzidenz handeln, zumal auch Einzelheiten in der Umgebung der Bande sich in Absorption und Emission entsprechen.

In früheren Arbeiten ${ }^{2,8}$ ist auf die auffallende Ähnlichkeit zwischen dem „blauen“ Spektrum des Benzaldehyds und dem Phosphoreszenzleuchten dieser Substanz hingewiesen worden. Das Auftreten des „blauen“ Spektrums von Benzaldehyd ist nach dem oben Gesagten jetzt durch eine Anregung der $\mathrm{C}=\mathrm{O}$ Gruppe im Benzaldehydmolekül bedingt. Dieser $\mathrm{Zu}$ stand hat in Absorption einen Extinktionskoeffizienten $\varepsilon=30$. Demgegenüber haben $\mathrm{Lew}$ is und $\mathrm{K}$ a s h a ${ }^{9}$ diesem Spektrum in Phosphoreszenz einen TriplettSingulett-Übergang des Benzaldehydmoleküls zugeordnet mit einer Lebensdauer des angeregten Zustandes von $0,1-0,01 \mathrm{sec}$. Die Lebensdauer, die aus dem Absorptionskoeffizienten gefolgert werden kann, ist

7 F. A l m a s y, J. Chim. physique 30, 537 [1937].

8 H. S c h ül e r u. A. W o e l d i k e, Z. Naturforschg. 1, 214 [1946].

9 G. N. L ew is u. M. K a s h a, J. Amer. chem. Soc. 66, 2100 [1944]; ebenda 67, 994 [1945]. sicher um einige Zehnerpotenzen kleiner, so daß diese beiden Auffassungen sich nicht ohne neue zusätzliche Annahmen miteinander vereinbaren lassen.

Vielleicht ist eine Erklärung in folgender Richtung zu suchen: Existiert unterhalb des Triplettzustandes eines Benzolderivates ein weiterer Anregungszustand, wie z. B. die $\mathrm{C}=\mathrm{O}$-Anregung der Aldehyde und Ketone, dann kann die in der Phosphoreszenz im Triplettzustand aufgespeicherte Energie an diesen tiefsten Zustand abgegeben werden, von wo aus der Übergang unter Emission zum Grundzustand erfolgt. Damit kann einerseits die Nachleuchtdauer der Phosphoreszenz durch die Lebensdauer des Triplettzustandes erklärt werden, während andererseits das beobachtete Leuchten der Phosphoreszenz nicht dem Übergang vom Triplettzustand zum Grundzustand entspricht. Vielmehr sehen wir jetzt einen Übergang, der nicht durch Multiplizitätsänderung verboten ist und daher auch in Absorption und bei Elektronenstoßanregung in der Glimmentladung beobachtet wird, nur daß bei der Phosphoreszenz dieser Zustand nicht direkt, sondern über den Triplettzustand besetzt wird.

Es wurde auf eine weitere Ähnlichkeit hingewiesen, die zwischen dem Phosphoreszenzleuchten und dem in der Glimmentladung beobachteten „blauen“ Leuchten von $p$-Xylol besteht. Nach unserer neuen Auffassung müssen wir nun dieses „blaue“ Spektrum bei Elektronenstoßanregung einer Verunreinigung durch $p$-Toluylaldehyd zuschreiben und nicht mehr dem aus $p$-Xylol gebildeten $p$-Tolylradikal. Damit erhebt sich nun die Frage, ob nicht auch das Phosphoreszenzspektrum des $p$-Xylols durch eine solche Verunreinigung vorgetäuscht sein kann. Für die Deutung des $p$-Toluylaldehydspektrums bei Phosphoreszenz, Elektronenstoßanregung und Absorption gelten in bezug auf die Lebensdauer des angeregten Zustandes die gleichen Überlegungen wie für Benzaldehyd.

Gegenüber dem Befund, daß das „blaue“ Spektrum auf eine gemeinsame Verunreinigung durch Benzaldehyd bzw. p-Toluylaldehyd zurückzuführen ist, haben wir in bezug auf das "grüne“ Spektrum, das bei $\mathrm{C}_{6} \mathrm{H}_{5} \cdot \mathrm{CH}_{3} ; \quad \mathrm{C}_{6} \mathrm{H}_{5} \cdot \mathrm{C}_{2} \mathrm{H}_{5} ; \quad \mathrm{C}_{6} \mathrm{H}_{5} \cdot \mathrm{C}_{3} \mathrm{H}_{7}(n)$; $\mathrm{C}_{6} \mathrm{H}_{5} \cdot \mathrm{CH}_{2} \cdot \mathrm{NH}_{2} ; \mathrm{C}_{6} \mathrm{H}_{5} \cdot \mathrm{CH}_{2} \cdot \mathrm{COOH}$ auftritt, auch bei sorgfältigster Reinigung keine Änderung in der Intensität feststellen können. Es handelt sich um das Spektrum, das, wie früher gezeigt ${ }^{2}$, nach dem Aussehen seiner scharfen Banden und dem Auftreten einer Hauptschwingung $\Delta v \sim 940 \mathrm{~cm}^{-1}$ dem Spektrum der ersten Anregung des Benzolringes ähnelt. Der Träger dieses gemeinsamen Spektrums läßt sich 
insofern schwer angeben, als Benzol außer diesem Spektrum noch eine weitere Emission im Grünen zeigt und Benzylcyanid $\left(\mathrm{C}_{6} \mathrm{H}_{5} \cdot \mathrm{CH}_{2} \cdot \mathrm{CN}\right)$ und $i$-Propylbenzol $\left(\mathrm{C}_{6} \mathrm{H}_{5} \cdot \mathrm{C}_{3} \mathrm{H}_{7}\right)$ zwar auch Bandenspektren im Grünen haben, die aber nicht mit dem von Toluol identisch sind. Die naheliegende Annahme, daß es sich um Übergänge zwischen höher angeregten $\mathrm{Zu}$ ständen handeln könnte, würde zunächst wohl die geringen Änderungen zwischen den „grünen“ Spektren beim Benzylcyanid, $i$-Propylbenzol und $z$. B. Toluol verständlich machen. Es ist aber dann schwierig, die Identität des „grünen“ Sprektrums bei Toluol und den erwähnten vier weiteren Monoderivaten des Benzols zu erklären, weil diese Substanzen bereits in dem Übergang vom ersten Anregungszustand zum Grundzustand deutliche Unterschiede zeigen, und man dann wohl nicht annehmen kann, daß solche Unterschiede bei höheren Anregungszuständen verschwinden sollten. Eine definitive Zuordnung dieser Spektren kann erst nach weiteren Versuchen gegeben werden.

Die Herstellung sowie die Reinigung der untersuchten Substanzen sind von Frl. Dipl.-Chem. R. Kö b erle durchgeführt worden.

\title{
Über den Solvatationszustand von Ionen in Flüssigkeitsgemischen
}

\author{
I. Leitfähigkeitsmessungen an Lithiumpikrat in Wasser-Äthanol-Mischungen \\ Von Gustav Kortüm und Albert Weller \\ Aus dem Physikal.-chem. Institut der Universität Tübingen \\ (Z. Naturforschg. 5 a, 451-456 [1950]; eingegangen am 25. Juli 1950)
}

\begin{abstract}
Die Leitfähigkeiten von Lithiumpikrat in Wasser-Äthanol-Gemischen werden in Abhängigkeit von der Salzkonzentration und vom Molenbruch des Lösungsmittels gemessen. Aus den Messungen werden durch Extrapolation auf unendliche Verdünnung die Äquivalentleitfähigkeiten $A_{\infty}$ ermittelt. Die daraus berechneten Grenzneigungen der $\mathrm{O}$ n s a g e $\mathbf{r}$ - Geraden zeigen erhebliche Abweichungen von den experimentell bestimmten Werten, selbst in Mischungen hohen Wassergehaltes. Aus diesen Abweichungen wird auf eine auswählende Solvatation der Ionen geschlossen. Im Zusammenhang mit der Ungültigkeit der W a ld e n schen Regel wird die Struktur solcher Wasser-Äthanol-Mischungen diskutiert.
\end{abstract}

\section{Problemstellung}

$\mathrm{D}$ er energetische und molekulare Zustand von reinen Flüssigkeiten und Flüssigkeitsgemischen bzw. Lösungen ist in neuerer Zeit der Gegenstand verschiedenster physikalisch-chemischer Untersuchungsmethoden geworden ${ }^{1}$. Wie aus diesen Arbeiten hervorgeht, liegt auch bei lückenloser Mischbarkeit der Komponenten in der Regel keine vollkommen homogene statistische Verteilung der Moleküle vor, sondern die Gemische zeigen eine ausgesprochene "Struktur", die einerseits durch die spezifischen zwischenmolekularen Kräfte, andererseits durch die sterischen Eigenschaften der Moleküle bedingt ist. Diese Struktur kann z. B. bei Mischungen von Alkohol mit Hexan oder von Phenol mit $\mathrm{CCl}_{4}$ durch eine

1 Vgl. z. B. K. L. W o lf u. Mitarbb., Z. physik. Chem. Abt. B 27, 58 [1934]; 36, 237 [1937]; 44, 359 [1939]. R. M e c k e u. Mitarbb., Z. Elektrochem. 52, 40, 49, 54, 269 [1948]; 53, 12 [1949]; Z. Naturforschg. 4 a, 183 [1949]. A. E u ck e n, Z. Elektrochem. 52, 255 [1948] und die dort angegebene Literatur. kettenförmige Assoziation der Alkoholmolekeln auf Grund von Wasserstoffbrücken gedeutet werden.

Bei Mischungen zweier polarer Komponenten läßt sich die Wechselwirkung der Partner im einzelnen bisher schwer übersehen. Für Äthanol-Wasser-Gemische, die in der Elektrolytforschung häufig als Lösungsmittel verwendet worden sind, sind die Möglichkeiten dieser Wechselwirkung auf Grund der makroskopischen Eigenschaften dieser Gemische früher diskutiert worden ${ }^{2}$. Der wesentliche Unterschied in der Struktur der beiden Komponenten liegt darin, daß in reinem Äthanol nur kettenförmige, also eindimensionale Assoziate über die H-Brücken möglich sind, während bei reinem Wasser eine lockere tridymitähnliche Schwarmstruktur ${ }^{3}$, nach der neueren Auffassung von Eucken ${ }^{4}$ neben Zweier- und

2 G. K o r t ü m, Z. physik. Chem. Abt. B 38, 1 [1937].

3 J. D. B ernal u. R. H. F ow le r, J. chem. Physics 1, 515 [1933]

4 A. E u c k e n, Göttinger Nachr. Math.-physik. Klasse 1946, 38; 1947, 20. 